\title{
Severe sclerokeratitis
}

due to Pseudomonas

\section{aeruginosa in non- contact-lens wearers}

\begin{abstract}
Purpose To review the clinical presentation, treatment and outcome in four cases of severe anterior segment infection by Pseudomonas aeruginosa unrelated to contact lens wear. Methods Four cases presenting over an 18 month period were reviewed.

Results The cases had variable presenting features and outcomes. Complications such as persistent infection, corneal thinning and phthisis bulbi were noted. Possible factors influencing adherence and tissue disruption are discussed.

Conclusions Suspicion of infection by $P$. aeruginosa and prompt isolation of the organism is needed early in the course of disease. Intensive and prolonged treatment with parenteral and topical antibiotics combined with judicious use of topical steroid gives the best chance of a favourable outcome.
\end{abstract}

Key words Conjunctiva, Cornea, Cefuroxime, Gentamicin, Pseudomonas aeruginosa

Pseudomonas aeruginosa infection of the eye can be severe and lead to significant permanent loss of vision. ${ }^{1,2}$ Consequences include perforation, scleritis, corneal scarring, corneal thinning, phthisis bulbi, cataract and persistence of infection. ${ }^{1-3}$ Multiple predisposing factors have been described, including advanced age, contact lens wear, pterygium surgery enhanced with mitomycin- $\mathrm{C}$ or beta radiation, cataract and glaucoma surgery, and trauma. ${ }^{3-7}$ We report four cases of severe ocular surface $P$. aeruginosa infection presenting to Manchester Royal Eye Hospital, each with a different and unusual predisposition.

\section{Case reports}

Case 1

An 80-year-old man with a history of myelodysplasia and psoriasis presented with spontaneous onset of a very large subconjunctival haemorrhage of the right eye. Best corrected vision at presentation was $6 / 9$ in the right eye and $6 / 6$ in the left. Ocular
R. RADFORD, A. BRAHMA,

M. ARMSTRONG, A.B. TULLO examination was otherwise unremarkable. Such was the swelling of conjunctival tissues and exposure that drainage of the subconjunctival haemorrhage with a sterile hypodermic 21 gauge needle was attempted in the emergency department. Treatment with Lacri-Lube ointment (Allergan) was given for exposure of conjunctival tissue. The following day the patient returned with a very painful red right eye, yellow discharge and suspected orbital cellulitis. On examination his visual acuity had worsened to $6 / 12$ in the right eye. He had conjunctival chemosis, superficial punctate keratopathy and bullae. The anterior chamber showed cells and fibrin and the pupil was fixed by posterior synechiae. The intraocular pressure (IOP) was $16 \mathrm{mmHg}$ and the posterior segment examination unremarkable. Haematological investigation revealed a mild pancytopenia: $\mathrm{Hb} 10.2 \mathrm{~g} / \mathrm{dl}$, WCC $2.1 \times 10^{9} / \mathrm{l}$, neutrophils $0.92 \times 10^{9} / 1$, platelets $102 \times 10^{9} / 1$ consistent with his myelodysplasia. Investigations to ascertain the nature and extent of infection were undertaken, including conjunctival swab, blood cultures, MSU, chest radiograph (old TB) and an orbital CT scan which indicated preseptal soft tissue swelling only. The patient was treated initially intravenously with metronidazole $500 \mathrm{mg}$ t.d.s. and cefuroxime $1.5 \mathrm{~g}$ q.d.s. and topically with gentamicin forte $1.5 \%$ and cefuroxime 5\% hourly.

By day 3 vision had deteriorated to hand movements (HM) despite clinical improvement in his cellulitis and reduced periorbital discomfort. The fundal view was obscured by anterior chamber activity and hypopyon. At this time the conjunctival swab was reported positive for $P$. aeruginosa and therefore intravenous therapy metronidazole was changed to gentamicin (cefuroxime was continued). Sensitivity of the isolate and of the other cases is shown in Table 1. By day 5 of admission there was a corneal ulcer and marked conjunctival necrosis had occurred (Fig. 1). Treatment was modified to g. prednisolone $0.5 \%$ q.d.s., g. ofloxacin 2 hourly and flurbiprofen $50 \mathrm{mg}$ b.d. orally. The intravenous cefuroxime $750 \mathrm{mg}$ q.d.s. was continued until day 7 when, with gradual clinical improvement
R.Radford

A. Brahma

A.B. Tullo

Department of

Ophthalmology

Royal Eye Hospital

Manchester, UK

M. Armstrong

Department of Microbiology Clinical Sciences Building

Central Manchester Health Trust

Manchester, UK

Mr R. Radford, MRCP, FRCOphth

Department of Ophthalmology

Royal Eye Hospital

Oxford Road

Manchester M13 9WH, UK

Tel: $+44(0) 1612766522$

Fax: +44 (0)161 2726618

Received: 19 January 1999 Accepted in revised form: 13 September 1999 


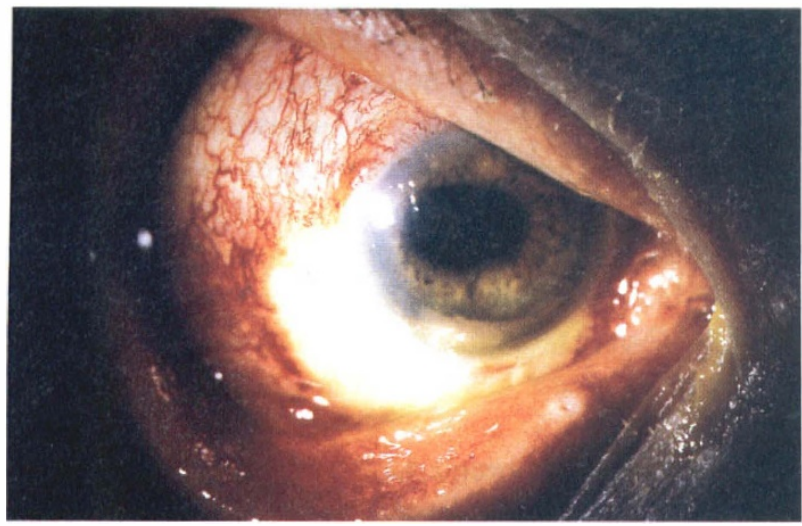

Fig. 1. Case 1. Right eye on day 5, showing marked conjunctival necrosis, corneal ulcer and hypopyon.

observed, the patient was discharged. After 1 month the vision had improved further to 6/18-1, the anterior segment continued to settle and the conjunctival defect was closing. A small area of inferior corneal thinning remained. Therapy was slowly tapered over the next 2 months and at 12 months after onset, vision was 6/9 with inferior corneal thinning.

\section{Case 2}

A 63-year-old man presented in September 1995 with a left retinal detachment when two retinal tears were found. He was bilaterally pseudophakic having previously had intracapsular cataract extractions with anterior chamber lens implantation elsewhere in 1990. The right eye was phthisical as a result of untreatable retinal detachment. He underwent surgery with vitrectomy and encirclement. Post-operative recovery was complicated by corneal decompensation and raised IOP, thought to be due to anterior segment ischaemia.

Treatment was commenced with g. prednisolone acetate $1 \% 2$ hourly, g. chloramphenicol $0.5 \%$ q.d.s., g. atropine $1.0 \%$ b.d. and g. levobunolol $0.5 \%$ b.d. Systemic acetazolamide $250 \mathrm{mg}$ b.d. and flurbiprofen $100 \mathrm{mg}$ t.d.s. were also given. He returned 7 days post-operatively with infiltrate causing complete corneal opacification of the left eye and signs of sclerokeratitis.

Surgery was undertaken, involving band removal, corneal scrape and a trephine biopsy for histology and microbiology. The initial report the same day found Gram-negative rods on the band but not from the corneal scrape. Ciprofloxacillin $750 \mathrm{mg}$ orally b.d. was commenced with topical gentamicin and ofloxacin alternate hourly. The corneal biopsy specimen confirmed $P$. aeruginosa. Despite initial therapy, increasing corneal melt occurred and the eye became soft. Based on antibiotic sensitivities of the organism ticarcillin was commenced, ofloxacin stopped and gentamicin forte q.d.s. continued. By day 14 of the infection there was no fundal view due to corneal opacity and vision had declined to perception of light. B-scan ultrasound demonstrated an attached retina. The antibiotic regime continued with the addition of oral tetracycline $500 \mathrm{mg}$ b.d. for 1 month. At review 21 days after onset $50 \%$ re-epithelialisation of the cornea had occurred. Areas of both corneal and scleral melt were observed. It was thought the condition of the cornea represented a combination of infectious keratitis and anterior ischaemia. One month after onset prednisolone $0.5 \%$ minims t.d.s. was commenced. At this stage the main findings were a thin opaque cornea with a persistent epithelial defect and an IOP of $3 \mathrm{mmHg}$. Penetrating keratoplasty and intraocular lens removal was performed 4 months after onset of infection. The graft remained clear but the eye became phthisical following repeated retinal detachment.

\section{Case 3}

A 72-year-old non-insulin-dependent diabetic man presented elsewhere with a 6 day history of sudden onset of a painful right eye. A thermal corneal injury to the same eye had occurred 40 years previously, involving hot ashes at a foundry. At presentation his visual acuity was recorded count fingers (CF) right and 6/6 left. A diagnosis of $P$. aeruginosa infection had been made from culture of a corneal scrape and treatment commenced with g. gentamicin forte, g. ciprofloxacin, oral amoxycillin $500 \mathrm{mg}$ t.d.s. and ciprofloxacin $750 \mathrm{mg}$ q.d.s. The clinical signs showed little change over the first 3 days and he was referred with a very red eye, conjunctival chemosis, $5.5 \mathrm{~mm} \times 4.5 \mathrm{~mm}$ corneal abscess with temporal ulceration, anterior chamber flare, cells, hypopyon and posterior synechiae. Treatment was adjusted to an intensive topical regime of $\mathrm{g}$. ciprofloxacin half-hourly, g. gentamicin half-hourly, g. atropine $1.0 \%$ b.d., oral amoxycillin and ciprofloxacin. After 6 days of intensive therapy there was a change of g. gentamicin to g. ofloxacin; g. prednisolone $0.5 \%$ b.d. was added. There was a steady and gradual improvement with settling of the anterior chamber inflammation and resolution of the corneal infiltrate. At the most recent follow-up 18 months after presentation there is residual corneal scarring and vision of $6 / 24$.

Table 1. Antibiotic sensitivities

\begin{tabular}{lllll}
\hline & Organism & Sensitivity & Resistance & Site \\
\hline Case 1 & Pseudomonas aeruginosa & $1,2,3,4,5$ & None recorded & Swab and corneal scrape \\
Case 2 & Pseudomonas aeruginosa & $1,2,5,6$ & None recorded & Band from retinal detachment surgery \\
Case 3 & Pseudomonas aeruginosa & $1,2,5$ & Not available & Corneal scrape \\
Case 4 & Pseudomonas aeruginosa & $1,2,3,5,6$ & 7,8 & Corneal scrape \\
\hline
\end{tabular}

1, ciprofloxacin; 2, gentamicin; 3, imipenem; 4, piperacillin; 5, ceftazidime; 6 , ticarcillin; 7, chloramphenicol; 8, cefuroxime. 


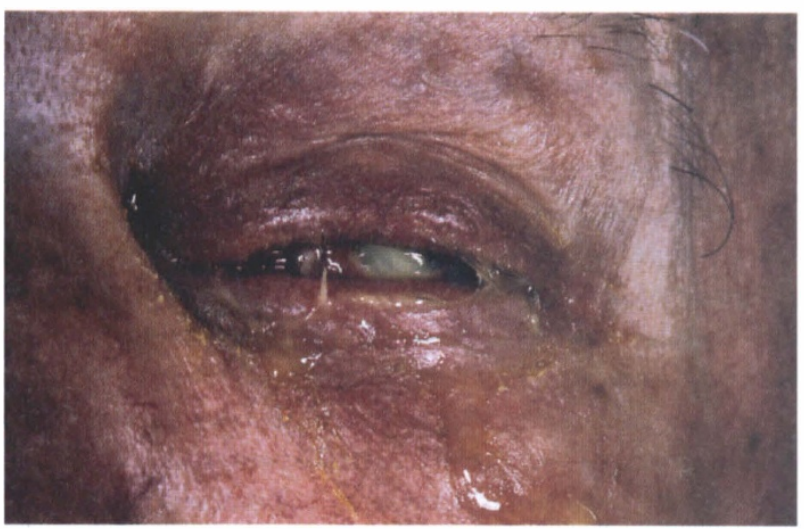

(a)

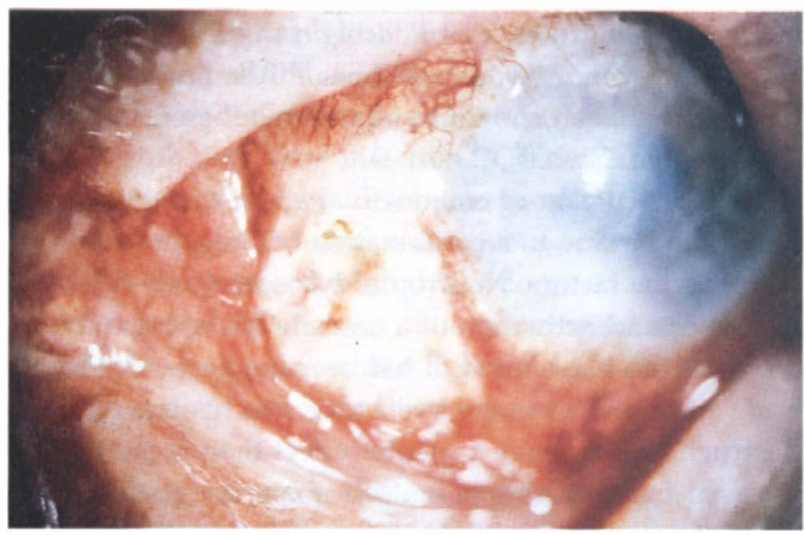

(b)

Fig. 2.a, b. Case 4. Left eye showing mucopurulent discharge, corneal abscess and hypopyon.

\section{Case 4}

A 75-year-old man attended following an apparently innocuous injury when cigarette ash was blown into his left eye. His eye became immediately painful which caused the patient to rub his eye intensively. He then washed his eye at the sink in a nearby public lavatory. An inferonasal conjunctival lump had been present in the eye for 20 years, which was the result of a foreign body injury when glass grinding with wax embedding. He was initially treated by his general practitioner for 3 days with ocular chloramphenicol. One week after the initial injury vision was 6/9 right, perception of light (PL) left. There was a copious mucopurulent discharge from the left eye (Fig. 2a). The conjunctiva was chemotic and prolapsing from the palpebral fissure. A corneal ulcer, abscess and $3 \mathrm{~mm}$ hypopyon were seen (Fig. 2b). A corneal scrape was taken and the patient admitted for intensive topical antibiotics, comprising g. gentamicin and g. cefuroxime. When $P$. aeruginosa was identified 1 day later oral ciprofloxacin was commenced and $g$. gentamicin was changed to g. ticarcillin. After a period of 8 days the patient was allowed home as his clinical signs had improved. However, he was readmitted with an inflamed eye and creamy white swellings present in the inferior nasal conjunctival quadrant at the site of the original lump 20 years prior. Exploration of the mass was undertaken, and histological examination revealed a

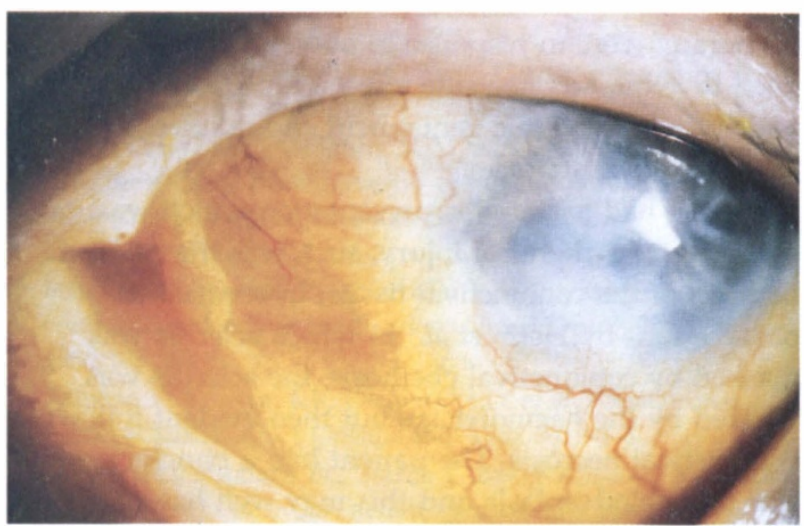

Fig. 3. Case 4. Residual scarring of the cornea.

granular eosinophilic material, histologically consistent with an abscess, though no growth was obtained. Following removal of this material the patient's eye quietened but he was left with a dense corneal scar (Fig. 3).

\section{Discussion}

$P$. aeruginosa is a ubiquitous organism, found on most domestic and hospital surfaces, and has even been isolated from presumed sterile containers including ocular medication. ${ }^{6,8}$ The organism can survive with the minimum of nutrition. ${ }^{9} P$. aeruginosa is not usually considered a commensal of the eye, but has been identified in normal conjunctival flora in less than $1 \%$ of cases. ${ }^{10} P$. aeruginosa is well described as an ocular pathogen, especially in association with contact lens wear, when epithelial trauma is thought to allow penetration through the ocular surface. Ocular infection is dependent on organism virulence, host immunity and site of infection. ${ }^{11} P$. aeruginosa sclerokeratitis is well recognised in elderly and debilitated patients. Alfonso et al. ${ }^{7}$ commented on the lack of coexisting systemic disease affecting these cases, although several diabetics are reported. More recently $P$. aeruginosa infection complicated by scleritis has been described following pterygium surgery with $\beta$-radiation or mitomycin $C$ application. ${ }^{3-5}$ In these cases a long period of latency is noted, suggesting either persistence of the organism, opportunistic infection of an altered surface, in particular bare sclera, or immune compromise of the irradiated surface. ${ }^{1,5}$ Once scleral invasion by $P$. aeruginosa has occurred it can be very difficult to eradicate. ${ }^{1,5}$ It is postulated the organism may reside between the avasular scleral lamellae and therefore be relatively remote from antibiotic attack. ${ }^{5,7}$

The four cases described show that $P$. aeruginosa may be the primary ocular pathogen in a variety of situations and in particular when recent or previous disruption to the ocular surface has occurred. In each of our cases the $P$. aeruginosa isolated was sensitive to gentamicin, ciprofloxacin, tircarcillin and ceftazidime. The choice of topical and systemic antibiotic was guided by the microbiologist's advice and patient suitability. Changes 
in therapy were in response to observed effect and the need to avoid adverse side effects, especially renal toxicity with systemic gentamicin and epithelial toxicity with topical gentamicin.

Case 1 had a dramatic presentation which was evocative of a chemical injury, such was the marked necrosis of the conjunctival tissue. In contrast to observations by Helm et al. ${ }^{1}$ in which scleritis was masked by conjunctival chemosis, the scleritis was masked by the haemorrhage but then evident following conjunctival necrosis. The patient had a panctyopenia due to myelodysplasia and this may well have contributed to both his large subconjunctival haemorrhage and subsequent infection. It is possible that after the needle puncture of the conjunctiva, the haemorrhage acted as a culture medium. It was also noted that this patient's scalp psoriasis improved markedly following intravenous antibiotics, though $P$. aeruginosa was not isolated from swabs of this site or from swabs of his topical psoariatic medication.

In case 2 the presence of a plomb is suggested as the nidus of infection. P. aeruginosa may exist in its own biofilm which can protect against antibiotic destruction and therefore perhaps also allow the organism to remain dormant. $^{12}$

Case 3 has a much less clear history and would suggest a true spontaneous onset of infection. Longstanding scarring existed in the cornea and one may speculate that this, together with his diabetic status, may have compromised the ocular surface defence.

Case 4 had several possible modes of infection. Inoculation with $P$. aeruginosa probably occurred as a consequence of ocular surface damage from vigorous rubbing plus irrigation with contaminated water. The conjunctival lump thought to be present following the previous foreign body injury and removed from the eye did not.harbour P. aeruginosa. The ocular inflammation, which subsequently settled, may have been of a chemical nature. Various surgical interventions are advocated in past reports and may help by removing bacterial load. ${ }^{1,7}$ It is possible in a structurally abnormal ocular surface that a biofilm such as that described in contact lenses may form around a focus of foreign material, in which organisms may reside and cause disease only after further compromise. ${ }^{13}$

The ocular surface has a variety of passive and active immune responses. The action of the eyelids and tearing provide the passive response and the following all have a role in preventing infection; secretory $\operatorname{IgA}(\mathrm{SIgA})$, complement, lysosyme, lactoferrin, betalysin, orosmucoid and caeruloplasmin, all of which decrease adhesion, may cause agglutination of the organism (thus rendering it non-virulent), or kill the organism by lysis of the bacterial cell wall. ${ }^{14}$ It is also said the normal flora of the conjunctival sac prevent more pathogenic bacteria proliferating. ${ }^{9}$ As a protective mechanism SIgA is produced and has been shown to inhibit the binding of P. aeruginosa. ${ }^{15}$ Immunisation of rabbit eyes to produce significantly raised levels of SIgA in their tears results in much less severe disease on subsequent exposure to
P. aeruginosa infection. ${ }^{15}$ The lacrimal gland contains a population of primed plasma cells and is therefore capable of producing antibodies against a variety of bacteria following bacterial contact with the common mucosal immune system of the gut and respiratory tract. ${ }^{16}$ It has been shown that corneal injury and duration of exposure significantly enhance the adherence of $P$. aeruginosa. ${ }^{17}$ It is postulated that cell injury either from direct trauma (induced in animal models), contact lens wear or resulting from the proteolytic agents released from $P$. aeruginosa itself, expose cell surface receptors which aid $P$. aeruginosa adherence. ${ }^{18}$ Protease IV has been shown experimentally to be directly toxic to corneal epithelium causing erosions and thus greatly enhances the virulence of $P$. aeruginosa when applied together with non-virulent strains. ${ }^{19}$ Alkaline protease has similary been shown to augment adherence to epithelial surfaces. ${ }^{20}$

The destruction of conjunctival and corneal tissue is known to be due to a combination of exogenous and endogenous factors. Neutrophil lysosomal release of proteases and activation of a cascade of destructive enzymes has been noted. It has been suggested by Steuhl et $a .^{20}$ that this 'innocent bystander' mechanism of tissue destruction may have a greater effect than factors released from $P$. aeruginosa itself. Experimentation in rabbit cornea has highlighted that it is the proteolytic neutrophil releases that contribute greatly to the corneal melt, especially in the initial stages of infection. ${ }^{20}$ Tissues also contain matrix metalloproteinases (MMPs), which are endopeptidases. MMPs can destroy almost any component of the extracellular matrix. Their activity is strictly regulated by gene expression, extracellular factors and tissue inhibitors. Experimentation has shown that features of $P$. aeruginosa infection can be inhibited or delayed by topical application of inhibitors of MMPs. ${ }^{21}$ In some recent work tear lipocalin has been identified as a cysteine proteinase inhibitor and may make up to $20 \%$ of tear protein. ${ }^{22}$ The relative proportion of this inhibitor and its presence in many other body fluids suggest it plays an important though yet unrecognised role in mucosal defence. The complex interaction and relative importance of the above individual factors is not clearly understood. ${ }^{15}$

It is now recognised that treatment of ocular $P$. aeruginosa infection involves control of the tissue destruction caused by the organisms' release of proteolytic enzymes, other tissue-damaging factors and suppression of destructive neutrophil lysosyme release. ${ }^{20}$ Current wisdom is to use topical and intravenous antibiotic therapy in the first weeks and only then to gradually institute steroid therapy. ${ }^{1}$ This is supported by previous work which showed that presumptive steroid use can be detrimental. ${ }^{21,23}$ Whilst specific guidelines are difficult with such a heterogeneous group of cases we make the following observations. Acute severe ocular surface infection requires prompt and broad spectrum antibiotic cover to rapidly reduce and eliminate infection We have until recently used intensive topical cefuroxime $5 \%$ and gentamicin $0.5 \%$ initially. This regime was 
modified in each case according to sensitivity and to avoid toxicity from prolonged gentamicin (seen after 4 days).

Ofloxacin is an excellent alternative topical agent providing broad antimicrobial cover, particularly against P. aeruginosa. ${ }^{24}$ Ofloxacin also has the advantages of low toxicity, prolonged tear film concentration, good penetration of cornea and aqueous and it comes ready to use in a preservative preparation for home use after discharge.

We highlight the potential complicating factors of spread and persistence of infection and feel intravenous therapy is warranted at the outset of treatment. This gives high concentrations in the surrounding adenexa (case 1 had preseptal cellulitis) and may give better scleral levels of antibiotic. It is also felt that combination intravenous treatment may reduce resistance - a major concern with persistent infection. ${ }^{1}$ Ceftazidime intravenously is known to achieve good intraocular concentrations and is shown to have excellent activity against $P$. aeruginosa. Aminoglycoside given with a third generation cephalosporin is synergistic against P. aeruginosa. ${ }^{1}$ Care is needed in the elderly or others with renal dysfunction and monitoring of levels is required.

For long-term oral treatment evidence suggests ciprofloxaxin provides good levels of ocular penetration although specific figures for intrascleral levels are again not available. ${ }^{25}$ Recommending a specific duration of therapy is difficult and the clinician should monitor the response to and side-effects of treatment when deciding when to stop antibiotics. Caution is suggested in view of the known scleral persistence of the organism and longterm antibiotic therapy is advocated. ${ }^{1,23}$

\section{Conclusion}

These cases highlight the need for careful history taking and examination, coupled with clinical suspicion that $P$. aeruginosa may cause severe anterior segment inflammation when there is no antecedent contact lens wear. Early diagnosis can be made by culture and microscopy of a conjunctival swab and/or corneal scrape. Prompt introduction and prolonged use of systemic and topical antibiotics are required followed by judicious use of topical steroid when clinical improvement is observed.

\section{References}

1. Helm CJ, Holland GN, Robert G, et al. Combination intravenous ceftazidime and aminoglycosides in the treatment of pseudomonal scleritis. Ophthalmology 1997;104:838-43.

2. Lotti R, Dart JKG. Cataract as a complication of severe microbial keratitis. Eye 1992;6:400-3.

3. Moriarty AP, Crawford GJ, McAllaster IL, Constable IJ. Severe corneoscleral infection: a complication of beta irradiation scleral necrosis following pterygium excision. Arch Ophthalmol 1993;111:947-51.
4. Rubifleld RS, Pfister RR, Stein RM, et al. Serious complications of topical mitomycin-C after pterygium removal. Ophthalmology 1992;99:1647-54.

5. Hsiao CH, Chen JY, Haung SCM, Ma HK, Chen PYF, Tsai RJF. Intrascleral dissemination of infectious scleritis following pterygium excision. Br J Ophthalmol 1998;82:29-34.

6. Schein OD, Wasson PJ. Microbial keratitis associates with contaminated ocular medications. Am J Ophthalmol 1988;105:361-5.

7. Alfonso E, Kenyon KR, Ormerod LD, et al. Pseudomonas corneoscleritis. Am J Ophthalmol 1987;103:90-8.

8. Schein OD, Hibbard PL, Starck T, Baker AS, et al. Microbial contamination of in-use ocular medications. Arch Ophthalmol 1992;110:82-5.

9. Pepose JS, Holland GN, Wilhelmus KR. Ocular infection and immunity. St Louis: Mosby, 1996:202-5.

10. Lochater-Khoranzo D, Gutierrez D. The bacterial flora of the healthy eye. In: Microbiology of the eye. St Louis: MosbyYear Book, 1977:14-7.

11. Allison JS, Dawson M, Drake D, Montie TC. Electrophoretic separation and molecular weight characterisation of Pseudomonas aeruginosa $\mathrm{H}$-antigen flagellin. Infect Immun 1985;49:770-4.

12. Davis DG, Parsek RM, Pearson JP, Iglewiski BH, Costerton JW, Greenberg P. The involvement of cell to cell signals in the development of bacterial biofilms. Science 1998;280:295-8.

13. McLaughlin-Borlace L, Stapleton F, Matheson M, Dark JK. Bacterial biofilm on contact lenses and storage cases in wearers with microbial keratitis. J Appl Microbiol 1998;84:827-38.

14. Dua HS. Bacterial keratitis in the critically ill and comatose patient. Lancet 1998;351:387-8.

15. Masinick SA, Montgomery CP, Montgomery PC, Hazlett LD. Secretory IgA inhibits Pseudomonas aeruginosa binding to cornea and protects against keratitis. Invest Ophthalmol Vis Sci 1997;38:910-8.

16. McClellan KA. Mucosal defence of the outer eye. Surv Ophthalmol 1997;42:233-46.

17. Ramphal R, McNiece MT, Polack FM. Adherence of Pseudomonas aeruginosa to the injured cornea: a step in the pathogenesis of corneal infections. Ann Ophthalmol 1981;13:421-5.

18. Gupta SK, Masinik SA, Hobden JA, Berk RS, Hazlett LD. Bacterial proteases and adherence of Pseudomonas aeruginosa to mouse cornea. Exp Eye Res 1996;62:641-50.

19. Engel L, Hill JM, Moreau JM, Green LC, et al. Protease IV produces corneal damage and contributes to bacterial virulence. Invest Ophthalmol Vis Sci 1998;39:662-5.

20. Steuhl KP, Doring G, Thiel HJ. Relevance of host derived and bacterial factors in Pseudomonas aeruginosa corneal infections. Invest Ophthalmol Vis Sci 1987;28:1559-68.

21. Kernacki A, Goebal MS, Hazlett P, Hazlett LD. Early TIMP gene expression after corneal infection with Pseudomonas aeruginosa. Invest Ophthalmol Vis Sci 1998;39:331-5.

22. Gachon A-MF, Lacazette E. Tear lipocalin and the eye's front line of defence. Br J Ophthalmol 1998;32:453-5.

23. Harben T. Recurrence of corneal Pseudomonas infection after topical steroid therapy: report of a case. Am J Ophthalmol 1964;58:670-4.

24. The Ofloxacin Study Group. Ofloxacin monotherapy for the primary treatment of microbial keratitis: a double-masked, randomised, controlled trial with conventional dual therapy. Ophthalmology 1997;104:1902-9.

25. Celebi S, Ay S, Aykan U, Bulut V, Alagoz G, Celiker UO. Penetration of oral and topical ciprofloxacin into human aqueous humor. Acta Ophthalmol Scand 1998;76:683-5. 